\title{
Intersection Graphs of Noncrossing Arc-Connected Sets in the Plane
}

\author{
Jan Kratochví1 ${ }^{1 \star}$ \\ Department of Applied Mathematics, Charles University, Prague, Czech Republic
}

\begin{abstract}
Arc-connected sets $A, B \subset E_{2}$ are called noncrossing if both $A-B$ and $B-A$ are arc-connected. A graph is called an NCAC graph if it has an intersection representation in which vertices are represented by arc-connected sets in the plane and any two sets of the representation are noncrossing. In particular, disk intersection graphs are NCAC. By a unified reduction we show that recognition of disk intersection and NCAC graphs are NP-hard. A simple observation shows that triangle-free disk intersection and NCAC graphs are planar, and hence recognizable in polynomial time. On the other hand, recognition of triangle-free $\mathrm{AC}$ graphs (intersection graphs of arc-connected sets) is still NP-hard.
\end{abstract}

\section{Intersection graphs}

Given a family of sets $\mathcal{M}$, a graph $G$ is called an intersection graph of $\mathcal{M}$ if a mapping $f: V(G) \rightarrow \mathcal{M}$ exists so that $f(u) \cap f(v) \neq \emptyset$ iff $u v \in E(G)$ for every pair of distinct vertices $u, v \in V(G)$. The multiset $\{f(u): u \in V(G)\}$ is called an $\mathcal{M}$ representation of $G$.

Intersection graphs, and namely intersection graphs of geometrical objects (elements of $\mathcal{M}$ are determined by their geometrical shape) were deeply studied both for their interesting graph theoretical properties and for their practical applications. Among these are interval graphs (intersection graphs of intervals on a line), circular arc graphs (intersection graphs of intervals on a circle), circle graphs (intersection graphs of chords of a circle), to mention just a few. These classes of intersection graphs are recognizable in polynomial time and allow polynomial algoritheorems for some otherwise NP-complete optimization problems.

Recently, practical applications led to introduction of more complex classes of intersection graphs, most of which are NP-hard to recognize. We mention grid intersection graphs (intersection graphs of vertical and horizontal grid line segments) $[5,11,3,1]$, box intersection graphs (intersection graphs of isothetic rectangles) $[15,17,14], S E G M E N T$ graphs (intersection graphs of straight line segments) $[4,12]$ or STRING graphs (intersection graphs of curves) $[16,4,9,10]$. At Graph Drawing '95, Breu and Kirkpatrick [2] considered disk intersection graphs and they presented a proof of NP-hardness of their recognition, both for the case of intersection graphs of unit disks, and of disks of bounded diameters.

\footnotetext{
* The author acknowledges partial support of Czech Research grants GA CR 0194 and
} GA UK 193 and of Czech-US Science and Technology Research grant No. 94051. 
The unbounded case remained open, and it is the aim of this note to show that recognition of disk intersection graphs (with no bound on the diameters of the disks) is NP-hard. Let us note that the technique of [2] is by its nature of no help for the case of unbounded diameters. Their technique also directly applies to touching graphs of bounded-diameter disks, while unbounded-diamater touching disk graphs are exactly planar graphs (cf. Koebe [8]) and hence easy to recognize. Thus the presented result is the last pebble in the mosaic of the complexity of variants of disk intersection and touching graph recognition problems. We will prove the result in a more general setting of noncrossing arc-connected sets in the plane.

\section{Noncrossing arc-connected sets}

For intersection graphs, it is important to distinguish between topological connectedness and arc-connectedness of sets in euclidean space. A set is called topologically connected if it cannot be split into two disjoint clopen subsets. On the other hand, a set is called arc-connected if any two of its points can be connected by a curve within the set (a curve is a homeomorphic image of a closed interval). It is well known [R.Thomas 1988 - unpublished personal communication] that every graph is an intersection graph of topologically connected sets in the plane. On the other hand, intersection graphs of arc-connected sets in the plane are exactly string graphs (intersection graphs of curves in the plane) $[16,10,4]$. These graphs are known to be NP-hard to recognize, but no finite recognition algoritheorem is known.

In some cases, the definitions of intersection graphs depend not only on the shape of the objects used to represent the vertices, but also on their relative position. E.g., grid intersection graphs fulfil the additional requirement that parallel segments of a representation are disjoint (and hence grid intersection graphs are always considered to be bipartite). Touching or contact graphs of segments, curves and disks attracted recently a lot of interest $[2,7]$. An important subclass of string graphs are 1-string graphs where no two curves of a representation are allowed to share more than one intersection point (recognition of such graphs is in NP). In this sense we introduce the following definition: We call two arcconnected sets $A, B$ noncrossing if both $A-B$ and $B-A$ are arc-connected. (Figure 1 shows an example of a crossing and noncrossing pair of sets.) In particular, any two disks are noncrossing, and more generally, any two homotopic convex sets are noncrossing. The impact of noncrossingness is illustrated by the following lemma, which will be implicitly used several times in the arguments in the next section.

Lemma 1. Let $A, B$ be noncrossing arc-connected sets inside a region $\Omega$. Let $a, a^{\prime}, b, b^{\prime}$ be disjoint curves in $\Omega$ such that $a, a^{\prime}$ start in $A$, end at the boundary of $\Omega$ and are both disjoint with $B$, while $b, b^{\prime}$ start in $B$, end at the boundary of $\Omega$ and are both disjoint with $A$. Then these curves meet the boundary of $\Omega$ in the cyclic ordering $a, b, b^{\prime}, a^{\prime}$ or $a^{\prime}, b, b^{\prime}, a$. 

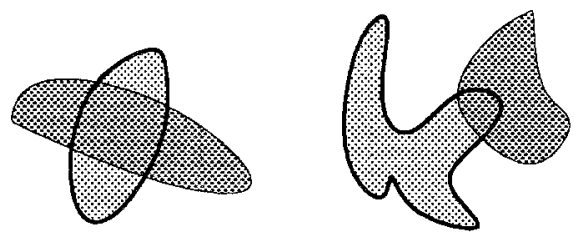

Fig. 1. Crossing and noncrossing pairs of sets.

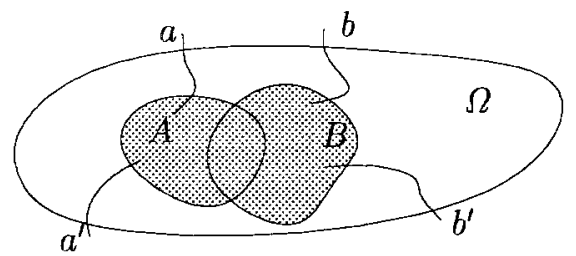

Fig. 2. Illustration to Lemma 1.

Proof. Denote $X_{a}$ a point in $a \cap A$ (and in a similar way, $X_{a^{\prime}} \in a^{\prime} \cap A, X_{b} \in b \cap B$, $\left.X_{b^{\prime}} \in b^{\prime} \cap B\right)$. Since $A-B$ is arc-connected, $X_{a}$ and $X_{a^{\prime}}$ can be connected by a curve $a^{\prime \prime}$ lying inside $A-B$. Similarly, $X_{b}$ and $X_{b^{\prime}}$ are connected by a curve $b^{\prime \prime}$ lying inside $B-A$. It follows that curves $a \cup a^{\prime \prime} \cup a^{\prime}$ and $b \cup b^{\prime \prime} \cup b^{\prime}$ are disjoint and both connecting two points on the boundary of $\Omega$. The Jordan curve theorem implies that the endpoints of $b \cup b^{\prime \prime} \cup b^{\prime}$ lie both on the same arc of the boundary of $\Omega$ determined by the endpoints of $a \cup a^{\prime \prime} \cup a^{\prime}$.

Lemma 2. Let $\mathcal{A}=\left\{A_{1}, A_{2}, \ldots, A_{n}\right\}$ and $\mathcal{B}=\left\{B_{1}, B_{2}, \ldots, B_{n}\right\}$ be systems of arc-connected sets such that

- every pair $A_{i}, B_{j}, i=1,2, \ldots, n, j=1,2, \ldots, m$ is noncrossing,

- every set $A_{i} \in \mathcal{A}$ intersects at most one set of $\mathcal{B}$ and vice versa, and

- both $G(\mathcal{A})$, the intersection graph of $\mathcal{A}$, and $G(\mathcal{B})$, the intersection graph of $\mathcal{B}$, are connected.

Then $A=\bigcup_{i=1}^{n} A_{i}$ and $B=\bigcup_{i=1}^{m} B_{i}$ are noncrossing arc-connected sets.

Proof. The union of non-disjoint arc-connected sets is arc-connected, and thus the (graph) connectedness of $G(\mathcal{A})$ implies the arc-connectedness of $A$ (and similarly for $B$ ).

Consider a set $A_{i} \in \mathcal{A}$. Since it is intersected by at most one set of $\mathcal{B}$, we have $A_{i}-B=A_{i}$ or $A_{i}-B=A_{i}-B_{j}$ for some $j \in\{1,2, \ldots, m\}$. In both cases, $A_{i}-B$ is arc-connected. Since every set of $\mathcal{B}$ intersects at most one set of $\mathcal{A}$, we have $\left(A_{i}-B\right) \cap\left(A_{j}-B\right)=A_{i} \cap A_{j}$ for every $i \neq j \in\{1,2, \ldots, n\}$. Thus the intersection graph of $\left\{A_{i}-B \mid i=1,2, \ldots, n\right\}$ is isomorphic to $G(\mathcal{A})$ and hence connected. It follows that $A-B=\bigcup_{i=1}^{n}\left(A_{i}-B\right)$ is arc-connected. $A$ 
similar argument shows that $B-A$ is arc-connected, and therefore $A$ and $B$ are noncrossing.

We define NCAC graphs as graphs with intersection representations by arcconnected sets in which any two sets of the representation are noncrossing. In particular, disk intersection graphs are NCAC graphs. We will prove in Section 3 that both recognition of disk intersection graphs and of NCAC graphs are NPhard problems. To get a better feeling about NCAC graphs and their NCAC representations, we state one more lemma here, which is a direct consequence of Lemmas 1 and 2 and which will be important in the proof of Theorem 4 (cf. the ladders in Fig. 4).

Lemma 3. Let $P_{1}, P_{2}$ be two paths such that every vertex of each of them is adjacent to at most one vertex of the other one. Suppose these paths have an $N C A C$ representation in a region $\Omega$ such that the sets representing the first and last vertex of $P_{1}$ intersect the boundary of $\Omega$ and the sets representing the inner vertices of $P_{1}$ as well as all vertices of $P_{2}$ lie inside $\Omega$. Then all sets representing vertices of $P_{2}$ lie in the same half-region determined by the chain of arc-connected sets representing $P_{1}$ and the boundary of $\Omega$.

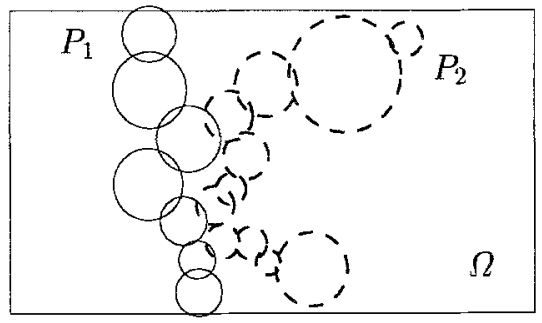

Fig. 3. Illustration to Lemma 3.

In other words, two chains of arc-connected sets cannot cross each other if sets in one of them are each allowed to intersect at most one set of the other chain. To enable a crosssing of chains, one set from one chain must be intersected by at least two sets of the other chain (cf. Fig. 5).

\section{Recognition of NCAC graphs}

We prove the main result in this section:

Theorem 4. Recognition of NCAC graphs is NP-hard. 

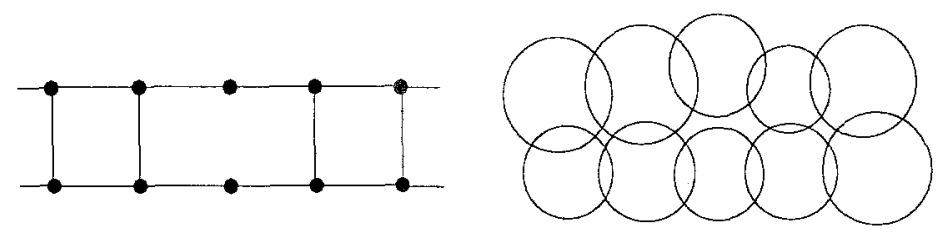

Fig. 4. A ladder.

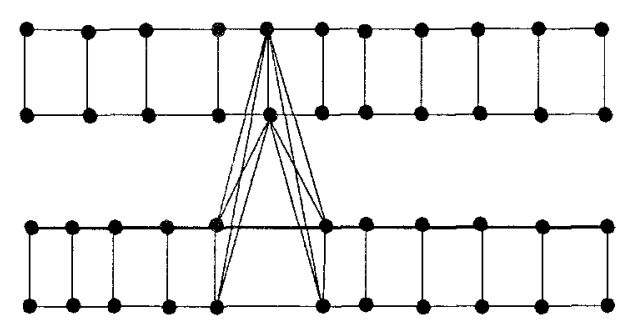

Fig. 5. Crossover component for two ladders.

Proof. We reduce from Not-All-Equal SATISFIABILITY. This is the problem

Instance: A CNF formula $\Phi=\bigwedge_{i=1}^{n} c_{i}$, where $c_{i}=\left(l_{i 1} \vee l_{i 2} \vee l_{i 3}\right)$ is a clause containing literals $l_{i 1}, l_{i 2}, l_{i 3}$ (a literal is a variable or a negation of a variable).

Question: Is there a truth assignment $\phi$ of the variables such that in every clause $c_{i}$, either one or two literals evaluate to TRUE?

Not-All-Equal SATISFIABILITY is known to be NP-complete even if all clauses contain exclusively positive literals (i.e., non-negated variables) and every variable occurs in exactly three clauses (this version is in fact exactly bicolorability of 3-regular 3-uniform hypergraphs) [6]. We assume that we are given such a formula $\Phi$ with a set of clauses $C$ over a set of variables $X$ (i.e., $C \subset\left(\begin{array}{l}X \\ 3\end{array}\right)$ ).

The reduction follows the guidelines of the reduction used in [9] to show the NP-hardness of recognition of string graphs.

We first embed the incidence graph $G_{\Phi}=(X \cup C,\{x c: x \in c \in C\})$ of $\Phi$ in a grid so that its vertices (both representing variables and clauses) are embedded in the grid vertices, and edges (connecting variables to clauses) are rectilinear, following the grid lines. Here comes the main difference from the construction in [9] - this embedding is not required to be noncrossing, in fact, it better should not be, as Not-All-Equal SAT is known to be solvable in polynomial time for formulas with planar incidence graphs. Note also that for such embedding it is important that $G_{\Phi}$ has maximum degree at most 4 (our $G_{\Phi}$ is cubic). We keep this embedding fixed.

Then we construct a graph $G$ from this embedding by local replacements. Each variable vertex will be replaced by a copy of the variable gadget, which 


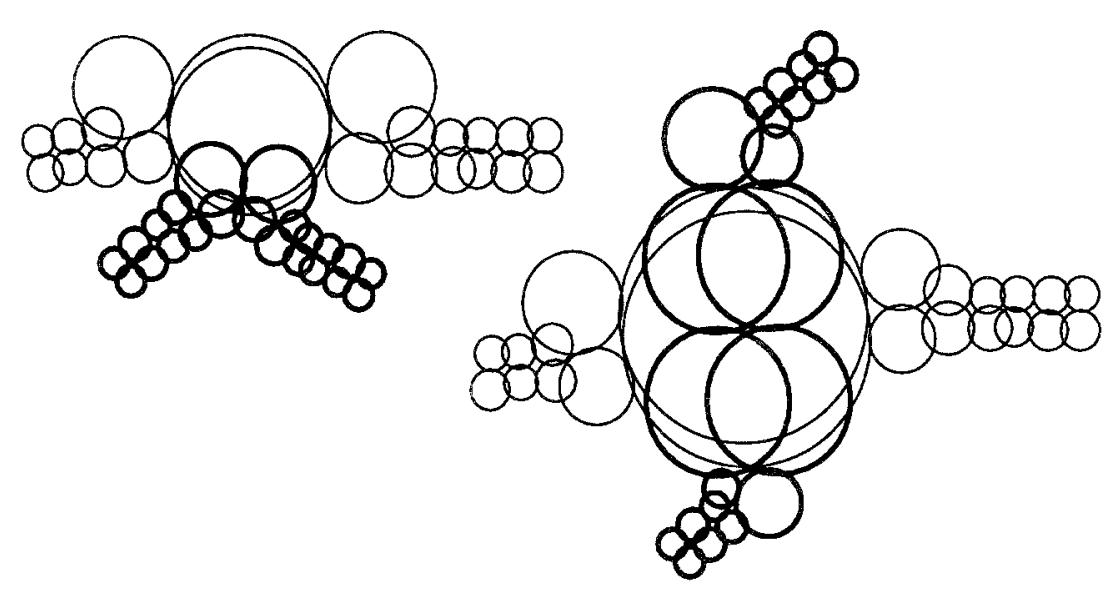

Fig. 6. Noncrossing and crossing realizations of a crossover component.

is simply a cycle of length 8 . Egdes of $G_{\Phi}$ are replaced by linking gadgets. In the reduction in [9], the linking gadgets were just pairs of parallel curves. The order of these pairs in which they arrive to the clause gadgets was used to encode whether the variable evaluates to TRUE or FALSE in the clause. In the presented reduction, the truth values will be encoded in the same way. The linking gadgets will be ladders (Fig. 4) of suitable length. A ladder is formed by two disjoint paths such that each vertex of each of them is adjacent to at most one vertex of the other path. Near each variable gadget, two ladders leaving that gadget are connected by a crossover component (Fig. 5). A crossover component allows two ladders to cross each other, if necessary, or to continue in their directions, whatever is necessary in a representation (Fig. 6). This is used when showing that an NCAC representation exists if $\Phi$ is Not-All-Equal satisfiable.

Every edge crossing in the drawing of $G_{\Phi}$ is also replaced by a crossover component. An essential argument in [9] was the topological uniqueness of the planar drawing of $G_{\Phi}$ (guaranteed by 3-connectedness of $G_{\Phi}$ ). In the present construction we force the topological uniqueness by inserting dummy connectors in $G_{\Phi}$, which are vertices inserted into faces of $G_{\Phi}$ and connected by chains to new crossover components placed on the edges of $G_{\Phi}$. (Details can be found in the technical report [13].)

Every clause is replaced by a clause gagdet depicted in Fig. 7. Unlike the gadgets used in [9], our clause gadget is non-symmetric. It requires a more detailed description. The clause gadget is bounded by a cycle of length 15 , and every fifth vertex is used for connection with the ladders bringing information from variable gagdets. Let this clause be $\nu=(x \vee y \vee z)$ and let the edges $x \nu, y \nu$ and $z \nu$ arrive to $\nu$ in this counterclockwise order in the fixed drawing of $G_{\Phi}$. We denote by $C_{x}, C_{y}, C_{z}$ the vertices of the clause gadget connected to the ladders, and by $l_{u}^{1}, l_{u}^{2}, r_{u}^{1}, r_{u}^{2}, u \in\{x, y, z\}$ the ladder vertices connected to 


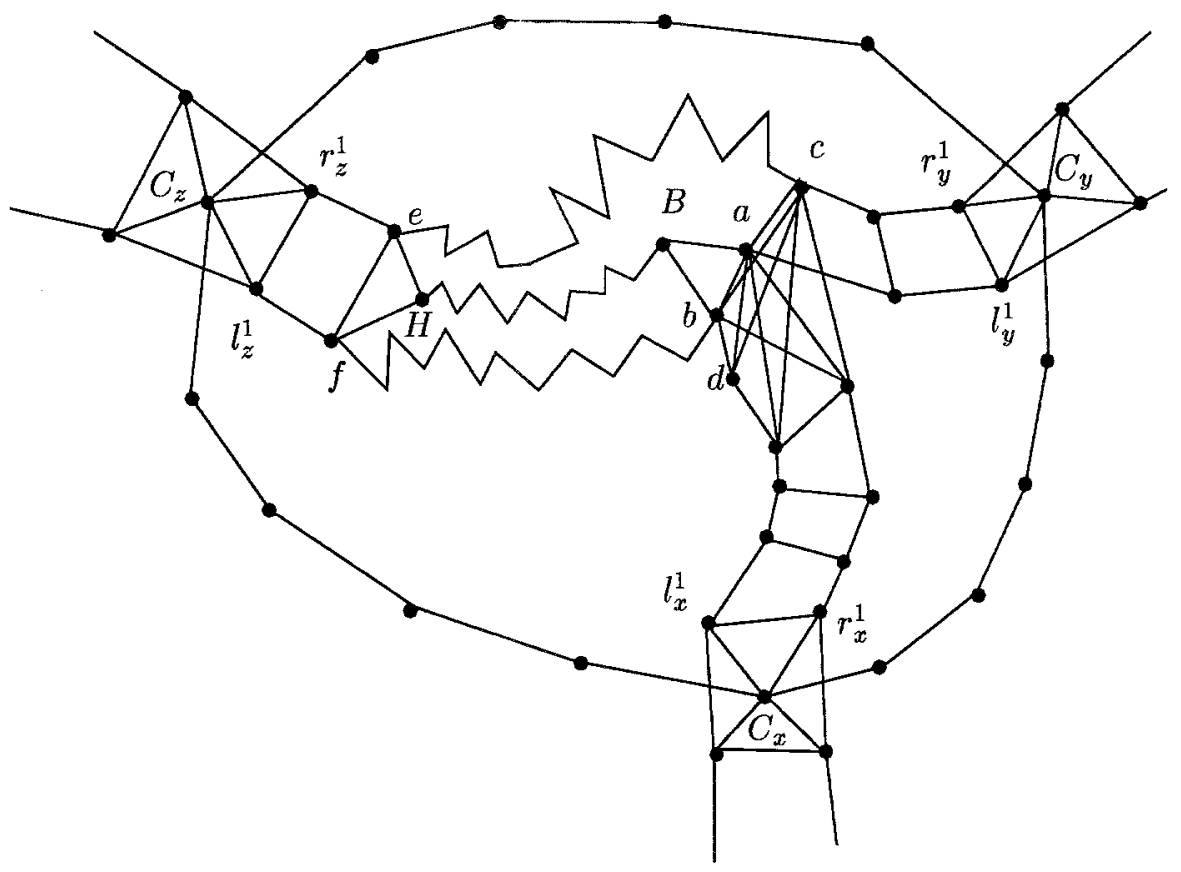

Fig. 7. The clause gadget.

$C_{u}, u \in\{x, y, z\}$. The ladders continue inside the clause-cycle, now as a part of the clause gadget. The $x$-ladder ends with vertices $b, d$, the $y$-ladder ends with $a, c$ and the $z$-ladder ends with $e, f$. The last four vertices of the $x$-ladder are pairwise adjacent to the last two vertices of the $y$-ladder. The inner part of the clause gadget is formed by vertices $B, H$ and by paths of suitable lengths (having a representation in mind, we call these paths chains): $B$ is adjacent to $a$ and $b$; $H$ is adjacent to $e$ and $f ; B$ is connected by a chain to $H ; c$ is connected by a chain to $e$ and $d$ is connected by a chain to $f$.

If $G$ has a representation by noncrossing arc-connected sets, then it has a representation such that the regions bounded by the cycles in variable gadgets are empty, and the regions bounded by the cycles in clause gadgets contain only the sets representing the vertices of the clause gadgets themselves. Contracting these regions into points and the ladders into edges, we obtain a drawing of $G_{\Phi}$. The dummy connectors force a topological uniqueness of this drawing and we may assume that this contraction is homotopic to the drawing we started with. In particular, in such a representation the ladders from variables to clauses arrive in the same circular order as in the drawing we started with.

The variable gadget can be represented in two different ways - its cycle being represented clockwise or counterclockwise. These two ways will encode if the variable is TRUE or FALSE. The ladders then transfer this information to 

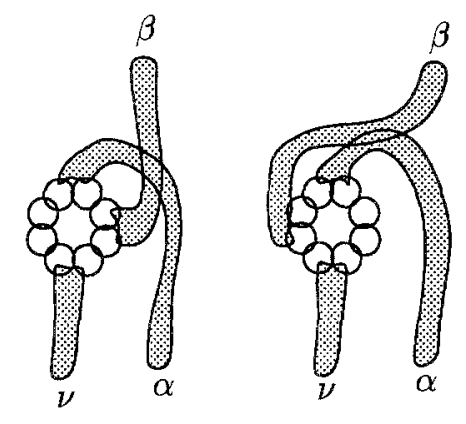

Fig. 8. Schematic picture of ladders leaving a variable occuring in clauses $\nu, \alpha, \beta$.
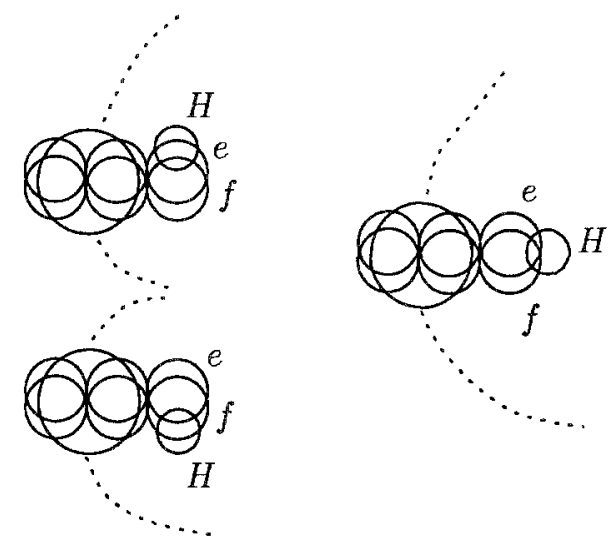

Fig. 9. Disk representations of $e f H$-part of a clause gadget.

the clauses - a variable, say $x$, is TRUE in a clause $\nu$ if and only if the sets $l_{x}^{1}, r_{x}^{1}$ arrive in this clockwise order to the clause gadget. In the notation of the masterclause $\nu=(x \vee y \vee z), x$ is TRUE in $\nu$ iff the sets $d, b$ appear on the boundary of the region bounded by the clause cycle in this clockwise order, $y$ is TRUE in $\nu$ iff $a, c$ appear in this clockwise order and $z$ is TRUE in $\nu$ if $f, e$ appear in this clockwise order.

Let us have a close look at a representation of the clause gadget. Suppose both variables $x, y$ evaluate to FALSE in $\nu$. Let $\Omega$ be the region inside the clause cycle, bounded by the sets representing the $x-, y$ - and $z$-ladders and the parts of the clause cycle between $C_{y}$ and $C_{z}$, and between $C_{x}$ and $C_{z}$. Since $B$ is connected to $H$ by a chain, the sets representing $B$ and $H$ must penetrate $\Omega$. We attend to the occurrence of $c, d, e, f$ on the boundary of $\Omega$. Since $c, a$ appear in this clockwise order and $B$ intersects $a$ but not $c$, the sets $c, B$ appear in this order. Similarly for $b, d$ ergo $B, d$. Hence $c, B, d$ appear in this clockwise order. 

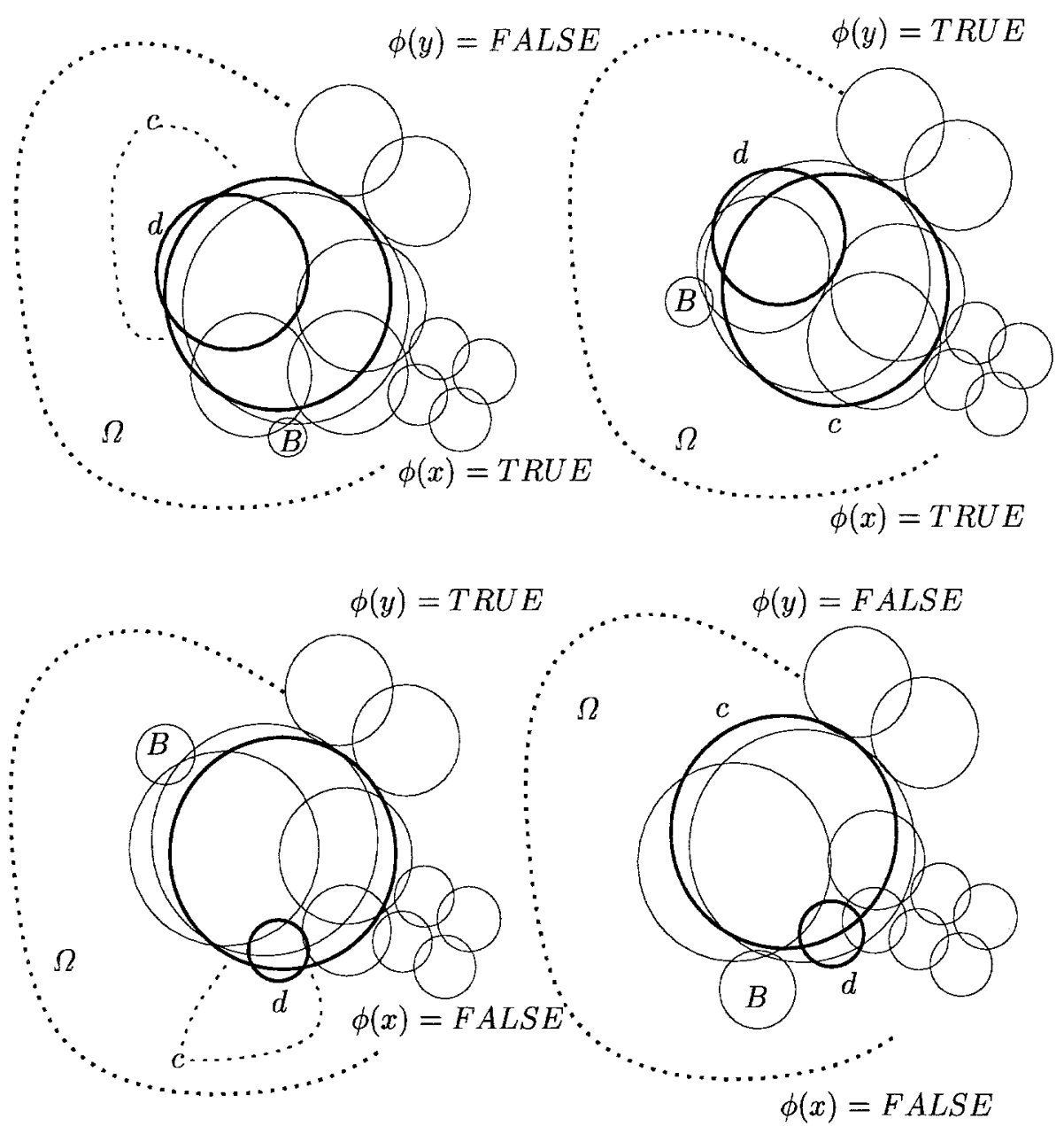

Fig. 10. Disk representations of $B c d$-part of the clause gadget.

Now the chains of arc-connected sets from $c$ to $e$ and from $d$ to $f$ satisfy the assumptions of Lemma 3 and therefore $f, e$ must occur in this clockwise order on the boundary of $\Omega$. This means that if $x, y$ are FALSE, $z$ must be TRUE. A similar argument shows that if $x, y$ are TRUE, $z$ must be FALSE. Thus we conclude that $\Phi$ is Not-All-Equal satisfiable, provided $G$ is an NCAC graph.

If, on the other hand, $\Phi$ is Not-All-Equal satisfied by a truth valuation $\phi$, an NCAC representation can be constructed along the same lines. We represent the variable gadgets clockwise or counterclockwise according to their truth values. The ladders will be realized along the edges of $G_{\Phi}$ in the drawing we started with. Near a variable, two ladders either cross or touch each other, in order to lead in the right direction (see schematic Fig. 8 and Fig. 6). So it remains to show that 


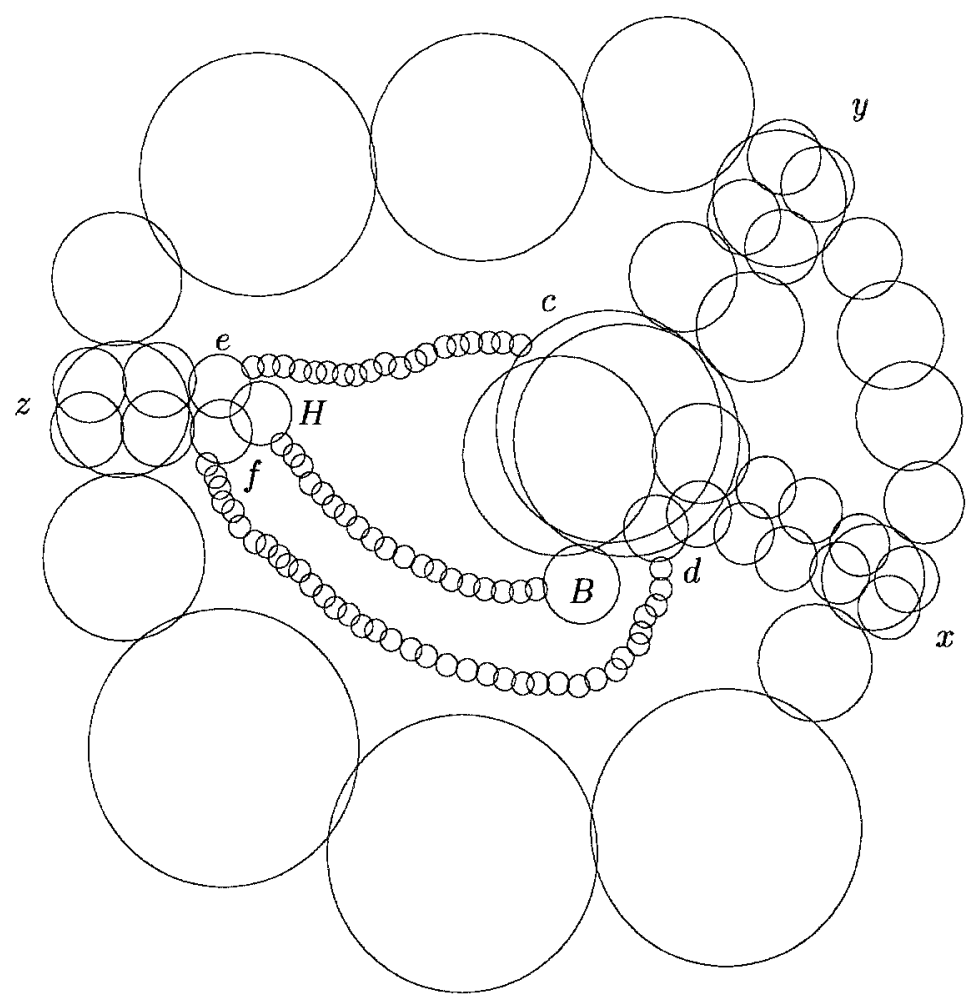

Fig. 11. Disk representation of clause gadget for $\phi(x)=\phi(y)=F A L S E$ and $\phi(z)=T R U E$.

the clause gadgets can be realized for all combinations of not-all-equal valuations of their variables. Figure 10 shows disk representations of the $x, y$-boundary of $\Omega$. It shows realizations of clockwise orders $c, a$ for $\phi(x, y)=\mathrm{FF}$ and $a, c$ for $\phi(x, y)=$ TT. It also shows that in case of $x, y$ being evaluated differently, both orders $a, c$ and $c, a$ are realizable. Figure 9 shows the $z$-boundary of $\Omega$ and it shows that all three relative positions of $H(e f H, e H f, H e f)$ are realizable. The realization of the entire gadget is then obvious, an example for $\phi(x, y, z)=$ FFT is show in Fig. 11.

Hence $G \in N C A C$ if and only if $\Phi$ is Not-All-Equal satisfiable, and $G$ is a disk intersection graph in such a case. Thus we have also proved the following corollary.

Corollary 5. Recognition of disk intersection graphs is NP-hard.

Note also that from the representations of particular gadgets, it would be possible to read a bound on the diameters (independent on $\Phi$ ) so that $G$ has a bounded-diameter representation whenever $\Phi$ is Not-All-Equal satisfiable. How- 
ever, the bounded-diamater case was completely treated in [2] and therefore we only stress the unbounded case here.

\section{Triangle-free graphs}

P. Hliněný [personal communication] noted that triangle-free disk intersection graphs are planar. This is true in the setting of noncrossing arc-connected sets as well:

Lemma 6. Triangle-free $N C A C$ graphs are planar.

Sketch of proof. Given a noncrossing arc-connected representation of a trianglefree graph $G$, choose a point in each $f(u) \cap f(v), u v \in E(G)$. Replace every set $f(u)$ by a dendrite (a connected union of curves not containing a closed curve) connecting the points in $f(u)$ so that the dendrites do not cross each other. This is possible since the sets themselves were noncrossing and their pairwise intersections were disjoint. One can easily deduce a planar drawing of $G$ from the dendrites.

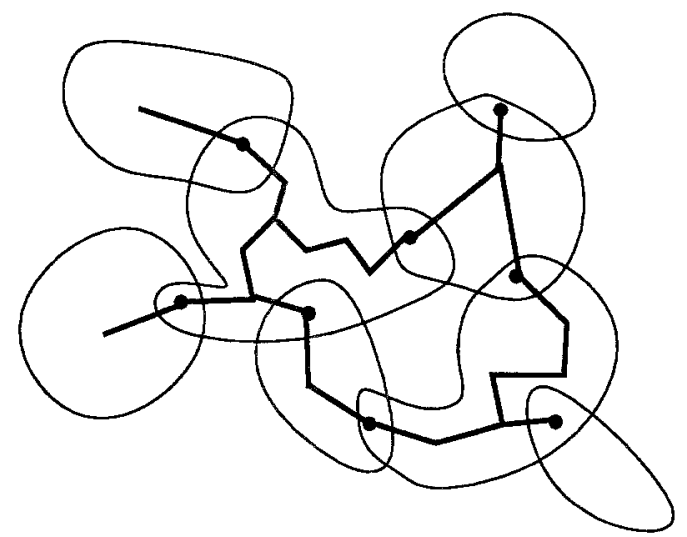

Fig. 12. Illustration to the sketch of proof of Lemma 6 .

Since every planar graph is a contact graph of disks [8], and thus a disk intersection graph, we see that triangle-free NCAC (and triangle-free disk intersection graphs) graphs are exactly triangle-free planar graphs. Therefore we conclude:

Corollary 7. Triangle-free disk intersection graphs, as well as triangle-free NCAC graphs, are recognizable in polynomial time. 
We should note that noncrossingness was crucial for the above observation. In general, traingle-free intersection graphs of arc-connected sets (triangle-free string graphs) are not necessarily planar - e.g., $K_{3,3}$ is a string graph. Also the recognition is more difficult:

Theorem 8. Recognition of triangle-free string graphs is NP-hard.

Proof. A reduction from Not-All-Equal SATISFIABILITY similar to the one presented above proves the result. We again start with a CNF formula $\Phi$ which has 3 non-negated variables in each clause and in which every variable occurs in 3 clauses. We fix a drawing of the incidence graph of $\Phi$ and add dummy connectors to enforce topological uniqueness of the drawing. Then we replace variable vertices by variable gadgets consisting of cycles of length 12 , connected to linking gadgets by every other vertex. Linking gadgets are pairs of induced paths (i.e., vertices from different paths are nonadjacent). Crossover components are edges of $K_{2,2}$ between pairs of vertices from two linking gadgets. Clause gadgets are slightly more complicated than in the reduction in [9] and in some sense resemble the gadgets from the previous section. The clause gadget is depicted in Fig. 13. The graph $G$ constructed in this way is triangle-free and we claim that it has an intersection representation by curves (and hence by arc-connected sets) if and only if $\Phi$ is Not-All-Equal satisfiable.

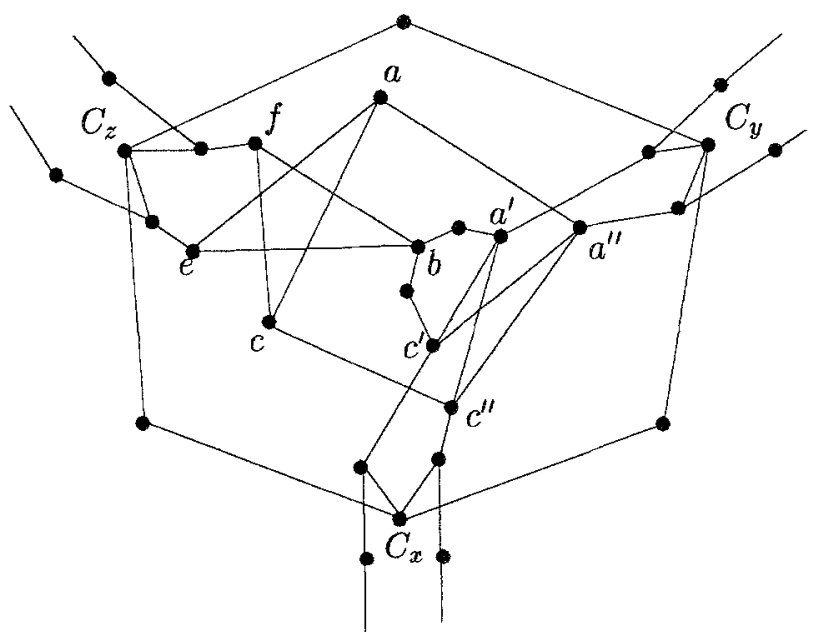

Fig. 13. The clause gadget for triangle-free string graphs.

Again, variable gadgets have two possible ways of representation - the variable-gadget cycle being represented clockwise or counterclockwise. These two ways are used to encode the truth values of the variables. This encoding is such 
that in the masterclause $\nu=(x \vee y \vee z), x(y, z$, respectively) is TRUE iff the curves $e^{\prime \prime}, c^{\prime}\left(a^{\prime}, a^{\prime \prime} ; f, e\right.$, respectively) lie in this clockwise order on the boundary of the region bounded by the clause-gadget cycle. Similarly as in the proof of Theorem 4, Table 1 shows feasible cyclic clockwise orderings of curves $a, b, c, e, f$ in a possible representation by intersections of curves (arc-connected sets) depending on the truth valuations of the variables in a clause $\nu=(x \vee y \vee z)$. It is easy to check (and known from $[16,9]$ ) that the pentagon acfbe has a representation inside a region if and only the cyclic ordering of its curves on the boundary of the region does not coincide with the complementary 5-cycle. Therefore $G$ has an intersection representation by curves if and only if every clause receives at least one TRUE and least one FALSE variable.

\begin{tabular}{|c|c|}
\hline$\phi(x, y, z)$ & cyclic orders of $a, b, c, e, f$ \\
\hline \hline FFF & $a b c e f$ \\
\hline FFT & $a b c f e$ \\
\hline FTF & bacef, bcaef \\
\hline FTT & bacfe, bcafe \\
\hline TFF & $a c b e f, c a b e f$ \\
\hline TFT & $a c b f e, c a b f e$ \\
\hline TTF & cbaef \\
\hline TTT & cbafe \\
\hline
\end{tabular}

Table 1. Feasible cyclic orderings for particular truth valuations.

\section{References}

1. Bellantoni,S., Ben-Arroyo Hartman,I., Przytycka,T., Whitesides,S.: Grid intersection graphs and boxicity, Discrete Appl. Math. (1994), 41-49

2. Breu, H., Kirkpatrick, D.: On the complexity of recognizing intersection and touching graphs of disks, In: Graph Drawing (F.J.Brandenburg ed.), Proceedings Graph Drawing '95, Passau, September 1995, Lecture Notes in Computer Science 1027, Springer Verlag, Berlin Heidelberg, 1996, pp. 88-98

3. Chandramouli,M., Diwan,A.A.: Upward numbering testing for triconnected graphs, In: Graph Drawing (F.J.Brandenburg ed.), Proceedings Graph Drawing '95, Passau, September 1995, Lecture Notes in Computer Science 1027, Springer Verlag, Berlin Heidelberg, 1995, pp. 140-151

4. Eherlich, G., Even, S., Tarjan, R.E.: Intersection graphs of curves in the plane, J. Combin. Theory Ser. B 21 (1976), 8-20

5. de Fraysseix, H., de Mendez, P.O., Pach, J.: Representation of planar graphs by segments, Intuitive Geometry, Colloquia Mathematica Societatos Janos Bolyai 63 (1991), 109-117 
6. M.R. Garey and D.S. Johnson, Computers and Intractability, W.H.Freeman and Co., 1978

7. P.Hliněný: Contact graphs of curves, In: Graph Drawing (F.J.Brandenburg ed.), Proceedings Graph Drawing '95, Passau, September 1995, Lecture Notes in Computer Science 1027, Springer Verlag, Berlin Heidelberg, 1996, pp. 312-323

8. Koebe,P.: Kontaktprobleme den konformen Abbildung, Berichte über die Verhandlungen der Sächsischen Akademie der Wissenschaften, Leipzig, Math.-Physische Klasse 88 (1936), 141-164

9. Kratochvíl,J.: String graphs I. There are infinitely many critical nonstring graphs, J. Combin. Theory Ser. B 52 (1991), 53-66

10. Kratochvíl,J.: String graphs II. Recognizing string graphs is NP-hard, J. Combin. Theory Ser. B 52 (1991), 67-78

11. Kratochvíl,J.: A special planar satisfiability problem and some consequences of its NP-completeness, Discrete Appl. Math. 52 (1994), 233-252

12. Kratochvíl,J., Matoušek,J: Intersection graphs of segments, J. Combin. Th. Ser. B 68 (1994), 317-339

13. Kratochvil,J.: Intersection graphs of noncrossing arc-connected sets in the plane (technical report), KAM Series, Charles University, 1996.

14. Kratochvíl,J., Przytycka,T.:Grid intersection and box intersection graphs on surfaces (extended abstract) In: Graph Drawing (F.J.Brandenburg ed.), Proceedings Graph Drawing '95, Passau, September 1995, Lecture Notes in Computer Science 1027, Springer Verlag, Berlin Heidelberg, 1996, pp. 365-372

15. Roberts,F.S.: On the boxicity and cubicity of a graph, In: W.T. Tutte, ed., Recent Progress in Combinatorics, Academic Press, New York, 1969, pp. 301-310

16. Sinden,F.W.: Topology of thin film RC-circuits, Bell Systern Tech. J. (1966), 16391662

17. Wood,D.: The riches of rectangles, in: Proceedings 5th International Meeting of Young Computer Scientists, Smolenice, 1988, 67-75 